\title{
Inhalt des Jahrganges 1975
}

Adamec, P., Diskussionsbeitrag

AUgrswald, H., Zur Rezeption sowjetischer Prosaliteratur im ersten Jahrzehnt der Deutschen Demokratischen Republik . . . . . . . . . . . . . . . . . . . . . . . . . 538

Auszüge aus Erzählungen Thomas Manns . . . . . . . . . . . . . . . . . . . . . . 664

BARtschat, B., Diskussionsbeitrag . . . . . . . . . . . . . . . . . . . . . . . . . 761

BAYER, E., Dimităr Blagoev und die Entwicklung der marxistischen Ästhetik und der proletarisch-sozialistischen Literatur in Bulgarien . . . . . . . . . . . . . . . . . . . . 545

BETrz, W., Die Sowjetliteratur der 60er Jahre als Literatur der entwickelten sozialistischen Gesellschaft . . . . . . . . . . . . . . . . . . . . . . . . . . . . . .474

Bielfeldt, H. H., Die Entlehnungen des Sorbischen aus dem Deutschen im 16. Jahrhundert 303

Bodola Y, G., Das ungarische Drama nach 1945 . . . . . . . . . . . . . . . . . . . 582

Бондарко, А. В., Проспект функщиовальной морфологии русского язнка . . . . . . . . . 726

BUDAGOVA, L. N., Von der antifaschistischen zur sozialistischen Literatur . . . . . . . . 258

Conrad, H., Nationales und Soziales in ihrer internationalen Bedeutsamkeit . . . . . . . 519

Craknko, E., Das Schaffen Adam Mickiewicz' in der Sowjetunion . . . . . . . . . . . . 24

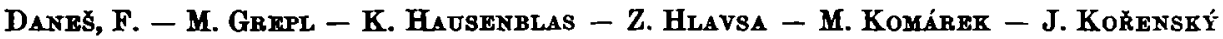
- R. MaizkK, Projekt einer theoretischen Konzeption der tschechischen Grammatik . . 613

DIIL, H.-O., Die Herausbildung der sozialistischen Nationalliteratur Kubas . . . . . . . . 587

EndLer, D., 7ur Gestaltung des sozialistischen Menschenbildes in der zeitgenössischen bulgarischen Prosa . . . . . . . . . . . . . . . . . . . . . . . . . . . . . . . . 557

Ekgelke, V., Polnische Motive in der DDR-Literatur . . . . . . . . . . . . . . . . . 131

Frmydank, D., Die Himmelfahrtshomilie „Veselite sę nebesa“ des Exarchen Johannes und ihre griechisehen Vorlagen . . . . . . . . . . . . . . . . . . . . . 382

GeIssler, K., Neue DDR-Beiträge zu Theorie und Geschichte des sozialistischen Realismus 483 GrissLER, W., Georg Weerth und die Entstehung der sozialistischen Literatur . . . . . . . 594

Grokargva, R. St., Die erste Bulgarisch-deutsche Grammatik ． . . . . . . . . . . . . 402

GLADROW, W., Diskussionsbeitrag . . . . . . . . . . . . . . . . . . . . . . . 744

GöвNEr, R., Zu einigen Besonderheiten der künstlerischen Zeitgestaltung im Erzählwerk Ivan Senčenkos . . . . . . . . . . . . . . . . . . . . . . . . . . . . . . . . 208

GrEPL, M., Die semantisch modifizierenden Komponenten der Satzstrukturen . . . . . . . 631

-, Diskussionsbeitrag . . . . . . . . . . . . . . . . . . . . . . . . . . . . . . . 662

Grochowskx, M., Grundlagen der Klassifikation semantischer Verknüpfungen zwischen prä- 673 dikativen Ausdrücken . . . . . . . . . . . . . . . . . . . . . . .

Grunzer, F., Die Diskreditierung des sozialistischen Realismus durch die maoistischen Führer in der Volksrepublik China . . . . . . . . . . . . . . . . . . . . . . . . . . . 491

Gutsceke, I., Das Verhältnis von Mensch und Natur in der sowjetischen Gegenwartsliteratur 526

FAGEB, R., Die Evolution des literarischen Menschenbildes im Erzählschaffen Sergej Antonov8, Jurij Nagibins und Vladimir Tendrjakovs der 50er Jahre . . . . . . . . . . . . 214

HAUSEnBLAS, K., Diskusgionsbeiträge . . . . . . . . . . . . . . . . . . . . . . 721, 807

HEINE, L., Adam Mickiewicz und die sorbische Literatur . . . . . . . . . . . . . . . . 91

Hermane, A., Zum Geleit [Mickiewicz-Heft] . . . . . . . . . . . . . . . . . . . . . 1

-, Zur Aufnahme und Wirkung der Ubertragungen des „Pan Tadeusz" in deutscher Sprache . . . . . . . . . . . . . . . . . . . 75

-, Bauer und Bauernklasse in der Gestaltung der Literatur der VR Polen . . . . . . . . . 567

HEXELsCHNeID kR, E., Sowjetliteratur über den Großen Vaterländischen Krieg in der Sicht bürgerlicher Kritiker . . . . . . . . . . . . . . . . . . . . . . . . . . . . . . 173

Hов́́LЕK, K., К вопросу т. наз. грамматичесного стандарта . . . . . . . . . . . . . . 768

IvančEV, S., Diskussionsbeiträge . . . . . . . . . . . . . . . . . . . . . . . . 656, 802 IvIĆ, M., Theoretische und methodologische Aspekte des Begriffs der Kondensation . . . . 774 , Diskussionsbeiträge . . . . . . . . . . . . . 661, 718, 796, 807 
JähxicheN, M., Die Literatur des antifaschistischen Widerstandskampfes im Formierungsprozeß sozialistischer Literaturen

Jüvger, H., Emmanuil Kazakevič und die Gestaltung des Heroischen in der sowjetischen Epik der Nachkriegsjahre . . . . . . . . . . . . . . . . . . . . . . . . . . . .

-, Die Entwicklung der Literaturen sozialistischer Länder und die Methodologie der Literaturwissenschaft

KAČALA, J., Grammatische und semantische Aspekte der syntaktischen Kondensation . . 797

KAROLAK, Sr., Zur Charakteristik der prädikativen Ausdrücke . . . . . . . . . . . . . 682

Kasper, K., Das Motiv der historischen Verantwortung des Menschen in Erzählungen Grigorij Baklanovs, Viktor Astaf'evs und Evgenij Nosovs der 60er und 70er Jahre . . . . 188

Kirchner, P., Unbekannte poetische Zeugnisse antifaschistischen Widerstands und proletarischen Internationalismus aus der Westukraine . . . . . . . . . . . . . . . . . 242

KomáREK, M., Diskussionsbeitrag . . . . . . . . . . . . . . . . . . . . . . . . . . . . 722

KowaLSKI, E., Der ,Stille Don“" und seine neuen Widersacher . . . . . . . . . . . . . 153

KulczYck a-Sat,ont, J., Adam Mickiewicz und die Positivisten . . . . . . . . . . . . . 13

LASkowski, R., Bemerkungen zu den Wortarten . . . . . . . . . . . . . . . . . . 704

-, Diskussionsbeiträge . . . . . . . . . . . . . . . . . . . . . . . . . . . . 660,745

Latchindan, A., Vom Subjektiven zum Assoziativen - eine Richtung in der zeitgenössischen sowjetischen und DDR-Epik? . . . . . . . . . . . . . . . . . . . . . 512

LEKov, I., Diskussionsbeitrag ． . . . . . . . . . . . . . . . . . . . . . . . . . . 719

LEŠKA, O., Diskussionsbeitrag . . . . . . . . . . . . . . . . . . . . . . . . . . 659

Libera, Z., Adam Mickiewicz und die Tradition der polnischen Aufklärung . . . . . . . . . 2

Lopatin, V. V., Diskussionsbeitrag . . . . . . . . . . . . . . . . . . . . . . . . 714

Luther, E., Ubber einige Tendenzen in der gegenwärtigen bulgarischen Prosa mit Dorfthematik

MrchatK, F., Diskussionsbeiträge . . . . . . . . . . . . . . . . . . . . . . 648, 781, 801

Михневич, А. Е., Синтаксическая конденсация и типы полипредикативных структур . . 788

-, Diskussionsbeiträge . . . . . . . . . . . . . . . . . 712, 745, 802, 803, 807

Mierad, F., Sergej Tret'jakov und Bertolt Brecht . . . . . . . . . . . . . . . . . . 226

Mistrí, J., Ausdrucksmittel der Hypersyntax . . . . . . . . . . . . . . . . . . . . 643

Moser, S., Zur Frage der Ubertragung von Werken der polnischen Romantik . . . . . . . 83

MrÁzEK, R., Diskussionsbeitrag . . . . . . . . . . . . . . . . . . . . . . 771

Novorný, J., Diskussionsbeitrag . . . . . . . . . . . . . . . . . . 647

OLschowsky, H., Romantische Traditionen in der polnischen Lyrik des 20. Jahrhunderts . . 99

Pisarkowa, K., Möglichkeiten und Erfordernisse der linguistischen Textanalyse . . . . . . 698

REISs, W., Erweiterung der rezeptionsästhetischen Aktivität bei der Rezeption der Sowjet-

literatur in den 70er Jahren . . . . . . . . . . . . . . . . . . . . . . . . . . . 532

ReIssier, E., Zur Rezeption der russischen Romantik im zeitgenössischen Deutschland 34

RŰžı̌̌̌̌, R., O соотнотении синтаксиса и значения предложения . . . . . . . . . . . 746

Schadmann, G., Der schöpferische Mensch . . . . . . . . . . . . . . . . . . . . . 496

Scholze, D., Die Stellung von Jerzy Putraments Roman „Bołdyn" in der polnischen Prosa

über den zweiten Weltkrieg . . . . . . . . . . . . . . . . . . . . . . 263

SchUSTER-SEwc, H., O8. hnydom, (so)gleich, sofort', ns. ned ,dass.' und Verwandtes . . . . 364

SEEhase, I., Zu einigen Entwicklungstendenzen der tschechischen Gegenwartsliteratur . . . 676

Soall, P., Zur Semantik der Deprädikation . . . . . . . . . . . . . . . . . . . . . 782

SKORUPKA, ST., Umgangssprachliche und idiomatische Ausdrücke und Wendungen im „Pan

Tadeusz" von Adam Mickiewicz . . . . . . . . . . . . . . . . . . . . . . . . . 63

Šváb, M., Ein neu erkanntes Hussitenlied in einem Erfurter Kodex . . . . . . . . . . . . 391 
SwIECH, J., Die deutsche Sprache in der polnischen Okkupationslyrik . . . . . . . . . . . 274

Szymczar, M., Adam Mickiewicz' Schaffen und die Entwicklung der polnischen Literatursprache . . . . . . . . . . . . . . . . . . . . . . . . . 58

THUn, F., Zur Kriegsproblematik in Daniil Granins Reisebeschreibungen . . . . . . . . . 199

THUN, N., Wirkungsästhetische Aspekte in Konstantin Simonovs Stalingrad-Romanen . . . 143

Topoursska, Z., Semantische Interpretation und formale Anslyse von Nominalgruppen in ausgewählten Fragmenten der Prosa von Thomas Mann . . . . . . . . . . . . . . 668

-, Diskussionsbeiträge . . . . . . . . . . . . . . . . . . 718, 723, 803

Uư̌xÝ, O., Zum strukturellen Charakter des sog. prädikativen Instrumentals im Tschechischen . . . . . . . . . . . . . . . . . . . . . . . . . . 369

-, Zur Frage der Beziehungen zwischen den syntaktisch relevanten semantischen Prädikatsmerkmalen und der Kasussemantik im Tschechischen . . . . . . . . . . . . . . . . 638

Walter, H., Zur Polysemie finiter Verbformen im Bulgarischen und ihrer Monosemierung 752

W ALther, G., Tagung der Internationalen Kommission zum Studium des grammatischen Aufbaus der slawischen Sprachen . . . . . . . . . . . . . . . . . . . . . . . . . . 611

-, Diskussionsbeitrag . . . . . . . . . . . . . . . . . . . . . . . . . . . . . 758

Whrner, H.-G., Der polnische Aufstand von 1830/31 and die deutsche politische Lyrik 114

WoJcrk, M., Byrons Dichtung in ihrer Zeit . . . . . . . . . . . . . . . . . . 42

Ziegengeist, G. - E. KowaLSKI, Slawistische Forschung am Zentralingtitut für Literaturgeschichte der Akademie der Wissenschaften der DDR im Zeichen des 30. Jahrestages der Befreiung vom Faschismus . . . . . . . . . . . . . . . . . . . . . . . . . . . 135

ZmmermanN, I., Diskussionsbeitrag . . . . . . . . . . . . . . . . . . . . . . . . . . . . 804

\section{Rezensionen}

Актуальные проблемы русского словообрааования. I, II - besprochen von I. Ohnheiser 421

Бернштейн, С. Б., Очерк сравнительной грамматики славянских языков - besprochen von R. Eckert . . . . . . . . . . . . . . . . . . . . . . . . . . . . . . . . . 817

Бочаров, А. Г., Человек и воина - besprochen von N. Thun . . . . . . . . . . . . . . 296

Bond, A., German Loanwords in the Russian Language of the Petrine Period - besprochen von H. H. Bielfeldt . . . . . . . . . . . . . . . . . . . . . . . . . . . . . . 842

Этимологический словарь славянских языков. Вып. 1 - besprochen von H. SchusterSewc . . . . . . . . . . . . . . . . . . . . . . . . . . . 824

Гончаров, В. П., Звуковая органивация стиха и проблемы рифмы - besprochen von H. Peukert . . . . . . . . . . . . . . . . . . . . . . . . 4 431

Каравфилов, Е., Съвременност и литературни жанрове - besprochen von D. Witschew 298

Линдберг, Г. У. - А. С. Герд, Словарь названий пресноводных рыб СССР на языках народов СССР и европейских стран. - Wörterbuch Nutzfische - besprochen von M. Bausch . . . . . . . . . . . . . . . . . . . . . . . . . . . . . . . . . . 430 LoRentz, F., Pomoranisches Wörterbuch, Bd. III. Nachträge - besprochen von A. E. Suprun 406

Mistrik, J., Exakte Typologie von Texten - besprochen von H. Peukert

Polański, K., Slownik etymologiczny języka Drzewian Połabskich, zesz. $2-3$ - besprochen von H. Schuster-Sewc 
Русская детская литература - besprochen von G. Bahro . . . . . . . . . . . . . . 604

RUSSIA MEDIAEVALIS. Tomus I - besprochen von G. Sturm . . . . . . . . . . . 437

Serbska bibliografija - besprochen von H. Pohrt . . . . . . . . . . . . . . . . . . . 300

С.товарь русского языка XI - XVIІвв. Вып. I. - Указатель источников в порндке а.лфавита сокращенных обозначений - besprochen von R. Eckert . . . . . . . . . . . 834

ST Axg, CHr. S., Opuscula linguistica - besprochen von K. Gutschmidt . . . . . . . . . 844

Sтокноғ, W. A. L., The extinct East-Slowincian Kluki-Dialect - besprochen von F. Hinze 407

Золотова, Г. А., Очерк функционального синтаксиса русского языка - besprochen von

W. Gladrow . . . . . . . . . . . . . . . . . . . . . 416

\section{Tagungsberichte}

Christopi, W., Aktuelle Probleme der russischen Gegenwartssprache in der Lehrerbildung 814

Fey ERHERD, V., Wissenschaftliche Arbeitstagung „Entwicklungsprobleme der Literaturen der sozialistischen Länder" . . . . . . . . . . . . . . . . . . . . . 600

Greifenhagen, G., Gemeinschaftskonferenz mit slowakischen Literaturwissenschaftlern in Smolenice . . . . . . . . . . . . . . . . . . . . . . . . . . . 293

Heinisch, R., Internationale Arbeitstagung zu Problemen des Sprachvergleichs . . . . . . 810

PoHrT, H., Arnošt-Muka-Tagung an der Karl-Marx-Universität . . . . . . . . . . . . . 404

ReISs, W. - R. HAGER - I. SchäFrR, Wissenschaftliche Arbeitstagung „Der schöpferische Mensch - Mensch und Arbeitswelt in der Sowjetliteratur der Gegenwart" . . . . . . . 602

Warar, G., Symposium zu Problemen der sowjetischen Literatur . . . . . . . . . . . . 599

W'mlnat, H., Arbeitatagung „Probleme und Ergebnisse russisch-deutscher und deutsch-russischer vergleichender sprachwissenschaftlicher Untersuchungen" . . . . . . . 812 